\title{
AN INVESTIGATION OF THE HYDRODYNAMICS OF HYPERSONIC JETS IN ASTROPHYSICAL CONDITIONS
}

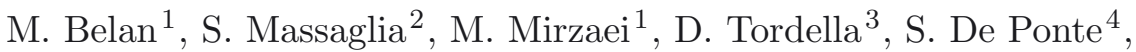 \\ A. Mignone ${ }^{2}$, A. Ferrari ${ }^{2}$ and E. Bodenschatz ${ }^{5}$
}

\begin{abstract}
Hypersonic, collimated jets are being lately intensively studied in Earth laboratories, trying to reproduce some of the physical properties of a subclass of astrophysical jets that are the Herbig-Haro $(\mathrm{HH})$ jets. These jets are produced in the regions around Young Stellar Objects (YSOs), that are proto-stars located inside galactic Giant Molecular Clouds. In addition to the novel experimental approach, $\mathrm{HH}$ or YSO jets have been object of interest by the astrophysical community since a few decades and studied by means of observations at different wavelengths and analytical and numerical modeling. We present laboratory experiments and 2D numerical simulations of hypersonic jets, comparing the results of experiments and simulations that reproduce the evolution of the above mentioned jets. The experimental flows match two main scaling parameter requirements for proto-stellar jets, i.e. the ejection Mach number $M$ and the jet/ambient density ratio $\eta$. In particular, $\eta$ goes from slightly underdense to overdense values. Furthermore, as a development of previous works, we consider here the dependence of the jet structure and morphology on the Mach number, in the range 10 to 15 .
\end{abstract}

\section{Introduction}

The study of YSO jets is carried out by the scientific community by means of direct observations at different wavelengths, MHD analytical studies, 2D and 3D

\footnotetext{
1 Dipartimento di Ingegneria Aerospaziale, Politecnico di Milano, Italy; e-mail: belan@aero.polimi.it

2 Dipartimento di Fisica, Università di Torino, Italy; e-mail: silvano.massaglia@unito.it

3 Dipartimento di Ingegneria Aeronautica e Spaziale, Politecnico di Torino, Italy; e-mail: daniela.tordella@polito.it

4 Retired, formerly Dipartimento di Ingegneria Aerospaziale, Politecnico di Milano, Italy

5 Max-Planck-Institute for Dynamics and Self-Organization, Goettingen, D; Georg August University, Goettingen, D; Cornell University, Ithaca, IL, USA;

e-mail: eberhard.bodenschatz@ds.mpg.de
} 
numerical simulations and laboratory experiments. Observations are typically carried out mainly in the optical and IR bands, but also in the radio and X-rays, and provide crucial diagnostic information on the jet physical parameters such as velocity, density, temperature and ionization fraction of the emitting matter, and magnetic field intensity (see contribution of T. Ray, this volume). The parameter space one derives from these observations sets the jet velocities in the range $100-400 \mathrm{~km} / \mathrm{s}$, and the Mach number between 10 and 40; the temperature and density of the emitting gas are typically about $10^{4} \mathrm{~K}$ and $10^{4}-10^{6} \mathrm{~cm}^{-3}$. The jet/ambient density ratio $\eta$, crucial parameter for investigating the jet propagation but rather difficult to constrain since the ambient is invisible on these scales, is estimated by indirect means between 1 and 10. With these parameters constrained, one can investigate, both analytically and numerically, the physical mechanisms that are responsible for the jet acceleration and collimation (Tzeferacos et al. 2009), and emission of line radiation (Teşileanu et al. 2008, 2009). Along with observations and analytical and numerical modeling, it is of primary importance to reproduce in the laboratory the physical processes characteristic of YSO jets, that mainly concern HD and MHD flows at hypersonic speeds. The literature reports results of experiments carried out in different laboratories that explore definite sets of jets parameters (Ciardi et al. 2009; Hartigan et al. 2009, also contribution of S. Lebedev, this volume). In Tordella et al. (2011) (Paper I) and Belan et al. (2011) (Paper II) we have focused our investigation on two crucial parameters that describe the hydrodynamics of YSO jets, i.e. the Mach number $M$ and the density ratio $\eta$. We have examined the propagation of these jets in the laboratory with $M \simeq 15$ and $\eta \simeq 1$ or $\eta>>1$. These results have been compared with those given by $3 \mathrm{D}$ numerical simulations for the same set of parameters. In the present paper, we present combined laboratory and numerical simulations about jets in the range $10 \leq M \leq 15$. The numerical simulations have been carried out in $2 \mathrm{D}$ axial symmetry, since the growth of non axial motions in the experiment appears only after the interaction between the jet and the end wall of the experimental chamber, what creates a backflow, and this event is not reached in the present work. In the experiment, the jets are produced by means of a fast piston coupled with de Laval nozzles, chosen from a set optimized for $5 \leq M \leq 20$. Different nozzles have very different output radii, so that the end wall is located respectively at 70 and 210 jet radii for the two nozzles used in this work.

\section{Experimental setup and numerical code}

The jets travel inside a modular vacuum vessel, with a maximum length of $5 \mathrm{~m}$ and a diameter of $0.5 \mathrm{~m}$, much larger than the diameter of the jets, so that the lateral walls effects are limited (Belan et al. 2008). The required ambient inside the vessel is obtained by means of a valves system that sets the ambient density, at pressures ranging from 1.5 to $100 \mathrm{~Pa}$, using a gas in general different from the jet gas. $\eta$ may range from 0.01 to more than 100 . The visualizations and measurements system is based on an electron gun equipped with a deflection system, which creates an electron sheet. This sheet intercepts the jet under 
test, generating a plane fluorescent section of the flow, which is imaged on an intensified camera. For each jet, it is possible to select a portion carrying out visualizations, density and velocity measurements on traveling structures (shocks, waves and large-scale eddies). Detailed descriptions of the experimental setup are available in Papers I and II. As done in those papers, we have employed the code PLUTO (Mignone et al. 2007) for the numerical calculations. As mentioned before, the simulations have been carried out on a $2 \mathrm{D}$ cylindrical domain in the coordinates $(r, z)$, normalized over the initial jet radius $r_{0}$ taken at the nozzle's exit. The cases $M=10$ and $M=15$ differ in the initial domain sizes because of the different de Laval nozzles adopted in the laboratory experiment. For $M=15$ the integration domain $R \times L$ extends on $7 \times 70$ jet radii and includes $140 \times 1400$ grid zones; the $M=10$ domain extends on $21 \times 210$ jet radii including $420 \times 4200$ grid zones. The boundary conditions are reflecting on the jet axis $(r=0)$ and at $z=L$, at $z=0$ we have the injection in the range $r=0$ to $r=1$ and free outflow outside. In the attempt to better mimic the actual experimental behavior of the fluid at the lateral walls of the vessel, the condition at $r=R$ sets the velocity to zero, differently from the condition in Papers I and II, where we allowed for partial reflection on the lateral boundary. The temporal behaviour of the flow injection into the domain reproduces the experimental one. For $M \simeq 15$ it is described in Paper I. For $M \simeq 10$ we have fitted the normalized flow rate as $Q=\theta\left(t_{1}-t\right) a_{0}\left[1-\exp \left(-a_{1} t\right)\right]+\theta\left(t-t_{1}\right) a_{2}\left[-\exp \left(-a_{3} t\right)+\exp \left(-a_{4} t\right)\right]$, where the $a_{i}$ coefficients and the piston final run time $t_{1}$ are related to the facility parameters, and $\theta$ is the step function. The next section reports an example of flow rate of this kind, see Figure 3.

\section{Results and conclusions}

We show numerical density distribution and experimental visualizations (light is proportional to the local gas density) at times when the jet heads cross the detection window. The numerical images show longitudinal cuts, exploiting the axial symmetry. The density is given in units of the unperturbed value in the vessel, before jet injection, while the time is given in units of the sound crossing time $\tau$ over the space unit, which is the nozzle exit radius $r_{0}$. A case of $M \simeq 15$ jet is illustrated in Figure 1, with the upper panel representing the density distribution at $t=16$ time units as resulting from the numerical calculations, while the lower panel shows the laboratory jet obtained from picture frames correlated as described in Paper I. The ratio $\eta$ at the nozzle exit is slightly greater than 1. Comparing numerical with experimental images, one notes the bright shape due to the density enhancement at the front bow-shock visible in both pictures, the density increase caused by the interaction with the lateral walls right behind, the bright knots along the jet that in the experimental image are not well resolved, and finally the lack of light emission from the low density region surrounding the jet. A $M \simeq 10$ jet having a density ratio similar to the jet of Figure 1 is shown in Figure 2. The jet morphology is similar to the one in Figure 1, but the size of the cocoon, if compared with the size of the jet core, is larger than in previous 


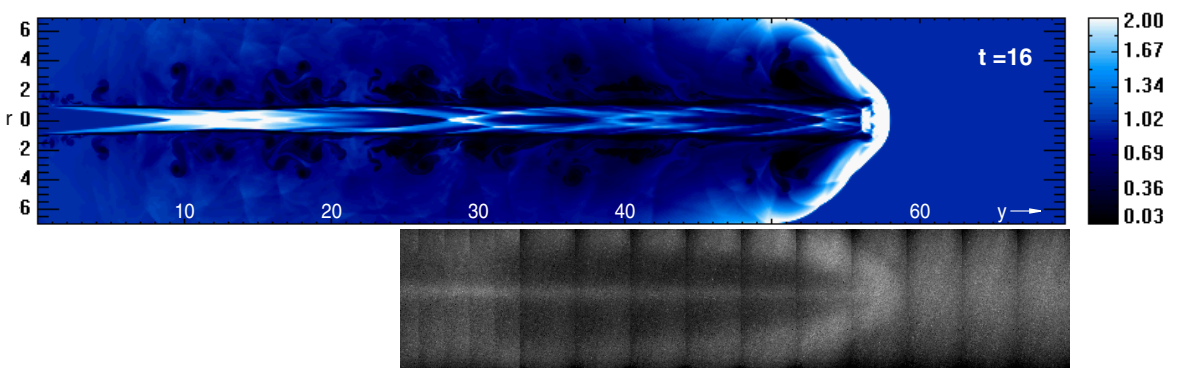

Fig. 1. Helium jet in Xenon, $M=15.3, \eta=1.4$. Space unit $r_{0}=35.7 \mathrm{~mm}$, time unit $\tau=0.23 \mathrm{~ms}$.

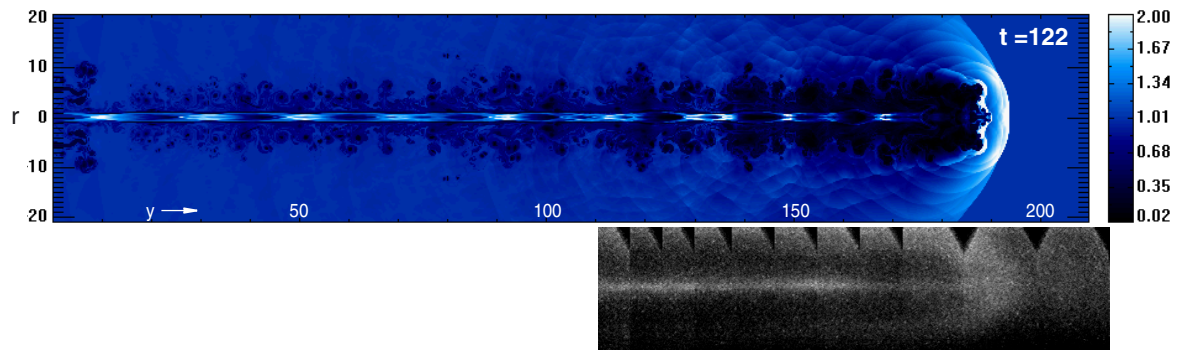

Fig. 2. Helium jet in Xenon, $M=10.9, \eta=1.2$. Space unit $r_{0}=12 \mathrm{~mm}$, time unit $\tau=69 \mu \mathrm{s}$.

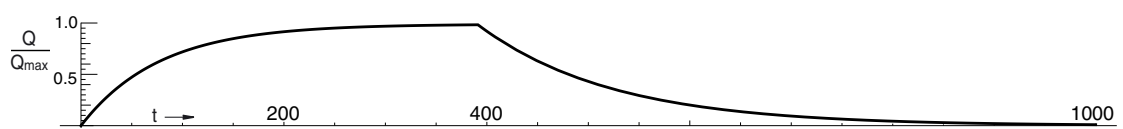

Fig. 3. Nondimensional flow rate vs. time for the jet of Figure 2.

case, as it can be expected for a lower Mach number. After taking into account the experimental uncertainties in imaging the density distributions, we find that observed morphologies and jet head velocities agree well with the ones from numerical simulations. An extended exploration of the parameter space with an extended discussion of the physics of the jet propagation will be the topic of a forthcoming paper.

\section{References}

Belan, M., de Ponte, S., \& Tordella, D., 2008, Exp. Fluids, 45, 501511

Belan, M., de Ponte, S., Tordella, D., et al., 2011, Ap\&SS, 336, 9 
Ciardi, A., Lebedev, S.V., Frank, A., et al., 2009, ApJ, 691, L147

Hartigan, P., Foster, J.M., Wilde, B.H., et al., 2009, ApJ, 705, 1073

Mignone, A., Bodo, G., Massaglia, S., et al., 2007, ApJS, 170, 228

Teşileanu, O., Mignone, A., \& Massaglia, S., 2008, A\&A, 488, 429

Teşileanu, O., Massaglia, S., Mignone, A., et al., 2009, A\&A, 507, 581

Tordella, D., Belan, M., Massaglia, S., et al., 2011, NJP, 13, 043011

Tzeferacos, P., Ferrari, A., Mignone, A., et al., 2009, Mon. Not. R. Astron. Soc., 400, 820 